\title{
Symptomatic cardiomyopathy as a presentation in Whipple's disease
}

\author{
PG de Takats, DLP de Takats, TH Iqbal, RDS Watson, MN Sheppard, BT Cooper
}

\begin{abstract}
Summary
A patient presenting with congestive cardiac failure and anaemia underwent investigation which led to the diagnosis of Whipple's disease, associated with dilated cardiomyopathy. Conventional antibiotic therapy for Whipple's disease resulted in resolution of the traditional features of Whipple's disease and a marked improvement in the patient's heart failure.
\end{abstract}

Keywords: Whipple's disease, dilated cardiomyopathy, endomyocardial biopsy

\section{Introduction}

First described in $1907,{ }^{1}$ valid cases of Whipple's disease reported in the world literature to date number around 800 . As originally suspected by Whipple, the condition is now considered to be of infective aetiology, ${ }^{2}$ although the nature of the infecting organism remains undefined. This rare multisystem disorder has a number of characteristics (see box). Cardiac involvement is unusual. Although a rarity, diagnosis of Whipple's disease carries great significance since effective treatment can prevent progression of an otherwise fatal outcome and instead result in cure.

Cardiological changes associated with Whipple's were first described by Upton in $1952 .{ }^{3}$ Previously, gross deformities of heart valves and the pericardium observed in post mortem cases were ascribed to co-existent rheumatic heart disease. However, more recent histological studies have shown periodic acid Schiff (PAS)-positive macrophages in the heart tissue of patients with untreated Whipple's disease, suggesting a direct relationship between the two entities. A variety of cardiac abnormalities are now recognised to occur in

\section{Department of}

Histopathology, Royal Brompton National Heart and Lung

Hospital, Sydney St, London SW3 6NP, UK MN Sheppard

Correspondence to Dr PG de Takats, Senior Registrar in Medical Oncology, Department of Clinical Oncology, Queen Elizabeth Hospital, Birmingham B15 2TH, UK
Whipple's disease, although cardiac involvement rarely gives rise to clinical manifestations and is usually an incidental post mortem finding. We describe a patient with clinical evidence of myocardial disease as a presenting feature of Whipple's disease, whose cardiac dysfunction responded to antibiotic therapy.

\section{Case history}

A 65-year old retired caucasian man was admitted to hospital with a six-week history of breathlessness and cough productive of white sputum, which had been getting progressively worse, despite digoxin and diuretic therapy from his general practitioner. No orthopnoea or ankle oedema was reported, but the patient felt non-specifically unwell, with arthralgia affecting small joints symmetrically, principally in the hands. His bowel habit was normal. Two years previously, he had lost one stone in weight over a period of months, which he had never regained. He was a retired engineer, married with no pets, having always lived in England and with no history of recent travel abroad. A life-long non-smoker, taking only modest quantities of alcohol, he had no other significant past medical history. His medication on admission comprised digoxin, coamilofruse, and ibuprofen.

On examination, he had a tanned complexion and was pyrexial, $37.5^{\circ} \mathrm{C}$. He had no jaundice, cyanosis, clubbing, or lymphadenopathy and was not obviously anaemic. He had a regular tachycardia of 120 beats/min with a gallop rhythm, but no murmurs were heard. His jugular venous pressure was not elevated and his chest was clear.

An electrocardiogram (ECG) showed atrial tachycardia with right bundle branch block and his chest radiograph was consistent with mild left ventricular failure. Initial blood results were as follows: haemoglobin $10.8 \mathrm{~g} / \mathrm{dl}$, mean corpuscular volume $66 \mathrm{fl}$, mean corpuscular haemoglobin $20.8 \mathrm{pg}$, white blood cell count $17.05 \times 19^{9} / 1 \quad(80 \%$ neutrophils $)$, serum albumin $29 \mathrm{~g} / 1$. Platelet count, plasma viscosity, serum B12, folate, biochemical profile and thyroid function tests were all normal. Faecal occult blood tests were negative, as were blood and sputum cultures.

Upper gastrointestinal endoscopy was performed to investigate the anaemia and revealed mild gastritis only. A small bowel biopsy showed normal villus architecture, but the lamina propria was infiltrated with PAS- 
positive macrophages diagnostic of Whipple's disease (figure 1). Antibiotic therapy was initiated and within 48 hours his fever and neutrophilia settled. He was discharged on daily tetracycline, iron supplements, and coamilofruse.

When reviewed in clinic three weeks later, the patient had developed exertional dyspnoea, orthopnoea, paroxysmal nocturnal dyspnoea, and ankle oedema, despite diuretic therapy. On examination, he was tachycardic, with a gallop rhythm and an ejection systolic murmur. His jugular venous pressure was now grossly elevated, with tender hepatomegaly and bilateral pitting oedema to his knees. A chest radiograph was consistent with worsening left ventricular failure, including a new small rightsided pleural effusion, while his ECG was unchanged. An echocardiogram showed dilatation of all four chambers of the heart, with generalised poor systolic function (figure 2A) and left ventricular ejection fraction (LVEF) estimated at $42 \%$.

The patient was treated for congestive cardiac failure with digoxin, diuretics, an angiotensin-converting enzyme (ACE) inhibitor, nitrates and hydralazine. On this treatment, he reverted to controlled atrial fibrillation and was therefore also anticoagulated with warfarin. He made a gradual improvement and when stable, coronary angiography and endomyocardial biopsy were performed. The coronary arteries were entirely normal and the LVEF was consistent with previous echocardiographic findings. Biopsies

A

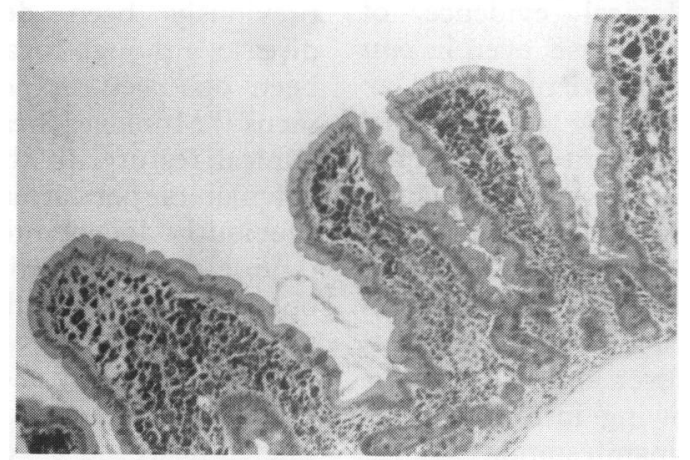

B

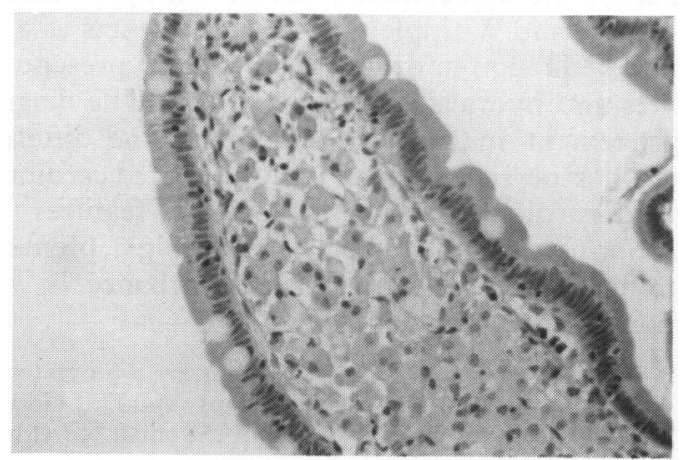

Figure 1 Small bowel biopsies confirming initial diagnosis of Whipple's disease. The extent of villus shortening and PAS stain uptake by macrophages is demonstrated in A $(\times 100)$, while at higher power, B $(X 200)$, numerous foamy macrophages with granular cytoplasm are visible within the villus from the left ventricle revealed interstitial fibrosis, variation in myofibre size, with hypertrophied as well as atrophic myocytes and myofibrillar loss (figure 3 ). There was also endocardial thickening with prominence of smooth muscle. A scanty lymphocytic infiltrate was also present in the interstitium. There was no evidence of pericardial disease and all arterioles were normal. Tests for amyloid were negative. No evidence of PAS-positive macrophages or bacillary bodies could be found at either light or electron microscopy. These histological changes were consistent with a clinical diagnosis of dilated heart muscle disease.
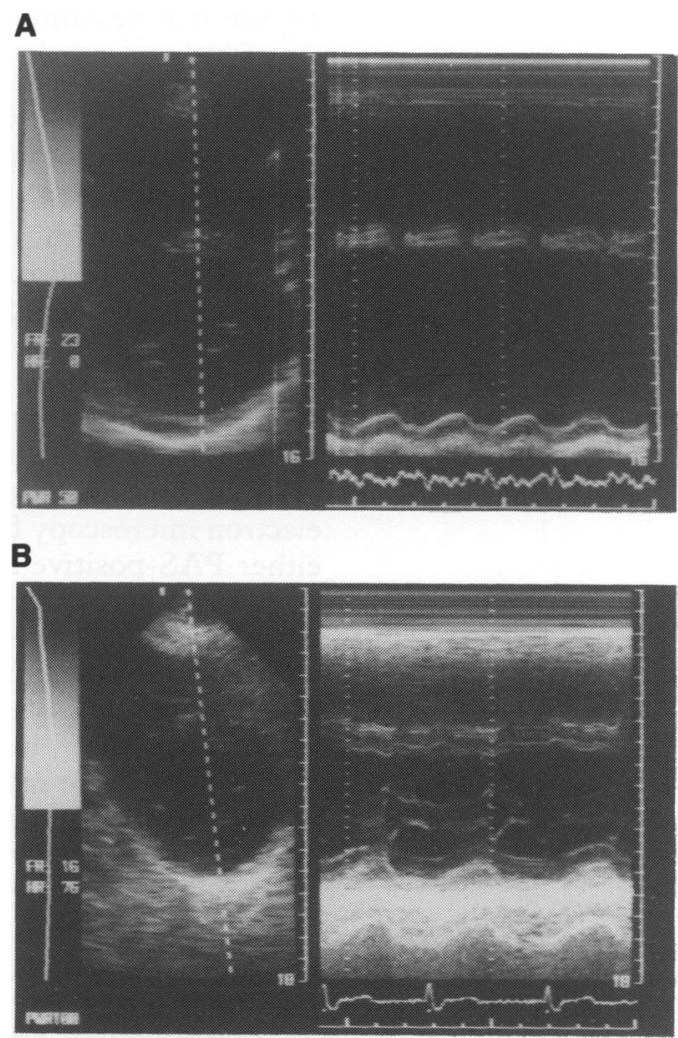

Figure 2 Echocardiograms performed (A) prior to and (B) after six months treatment for Whipple's disease, indicating a significant increase in left ventricular ejection fraction from $42 \%$ to $58 \%$ with treatment

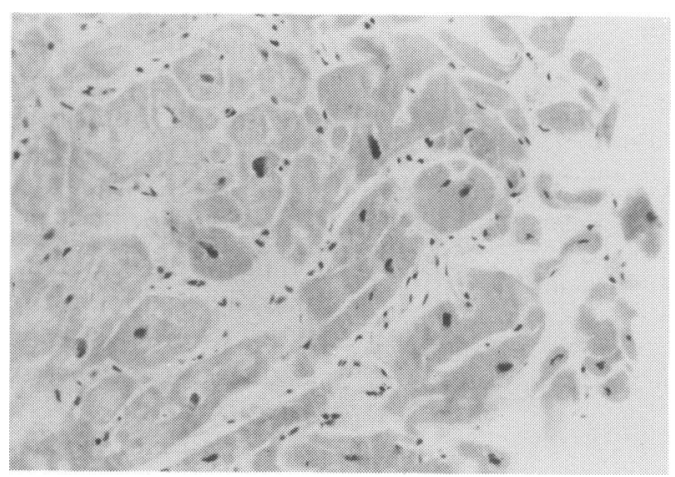

Figure 3 Endomyocardial biopsy showing irregular myocytes, some of which are hypertrophic with large nuclei while others are attenuated. Note also the myofibrillar loss in some of the myocytes. There is an increase in interstitial connective tissue (H and $\mathrm{E}$ stain, $\times 350$ ) 
Six months after starting antibiotic treatment, the patient's heart failure was well controlled, having discontinued nitrate and hydralazine therapy. His anaemia resolved, with replenished iron stores and his serum albumin normalised. Repeat small bowel biopsy failed to show any PAS-positive macrophages in an otherwise normal-looking specimen. At that time, he remained in controlled atrial fibrillation, and repeat echocardiography showed considerable resolution of cardiac hypertrophy, with significant improvement in LVEF, estimated at 58\% (figure 2B).

The patient is now two years from commencing antibiotic therapy and has reverted back to sinus rhythm. He remains on tetracycline, digoxin, and lisinopril, currently with no clinical manifestations of Whipple's disease.

\section{Discussion}

In the patient presented here, the close temporal association of the rapid development of heart failure with clinical and histological evidence of dilated cardiomyopathy and additional clinical manifestations of Whipple's disease strongly implies that the myocardial disorder was due to Whipple's disease. This view is supported by his response to therapy and subsequent recovery of cardiac function.

At the time of heart muscle biopsy, light and electron microscopy failed to show evidence of either PAS-positive macrophages or bacillary bodies in biopsy specimens. However, the patient had been on antibiotic treatment for six weeks prior to the biopsy. In addition, there is also the likelihood of sampling error, while it is recognised that histological evidence of Whipple's disease may be sparse, even in post mortem hearts of untreated patients. ${ }^{4}$

Three weeks after initiating antimicrobial therapy, the patient was admitted to hospital with florid signs of cardiac failure. This is not altogether surprising, since clearance of PASpositive macrophages from the gut is known to be tremendously variable, requiring several weeks to many years ${ }^{5}$ in some instances. Thus, while features such as fever and neutrophilia may settle rapidly following introduction of antibiotics, more severe manifestations such as cardiac dysfunction would be expected to be more sustained. In favour of the association between the myocardial disease and Whipple's in this case is that, despite a reduction in drug treatment (notably, nitrates and hydralazine), there was a major improvement in cardiac functional capacity, which has persisted over time. In idiopathic dilated cardiomyopathy, changes in ejection fraction over time are usually small and the significant increase from $42 \%$ to $58 \%$ over six months in this patient could not be attributed to ACE inhibitor therapy alone.

In post mortem studies on hearts of patients

1 Whipple GH. A hitherto undescribed disease characterised anatomically by deposits of fat and fatty acids in the intestinal and mesenteric lymphatic tissues. fohns Hopkins Hosp Bull 1907; 18: 382-91.

2 Relman DA, Schmidt TM, MacDermott RP, Falkow S. Identification of the uncultured bacillus of Whipple's disease. N Engl Med F 1992; 327: 293-300. dying from Whipple's disease, the pathological changes observed are consistent with that of a chronic inflammatory process. The pericardium is most commonly involved, with pericarditis, pericardial adhesions and/or pericardial effusions occuring in up to $60 \%$ of patients. ${ }^{1,6,7}$ Endocardial disease is a frequent finding, with incidental cardiac murmurs documented in up to $30 \%$ of patients during life, while post mortem reports describe valvular vegetations in at least $50 \%$ of cases. ${ }^{8}$ Myocardial involvement, however, is rare. There are only a few cases in the literature which describe myocarditis as part of a pancarditis, in an otherwise grossly normal heart, diagnosed usually at post mortem, ${ }^{7,9}$ but, on one occasion, during life. ${ }^{4}$ Myocarditis in the face of normal heart valves, chambers, and coronary vasculature has only once been previously documented and was an unexpected post mortem finding in a patient dying of Whipple's disease. ${ }^{10}$ Chronic damage to the pericardium, endocardium and, less frequently, the myocardium resulting from the polyserositis characteristic of Whipple's disease is probably the cause of death in most patients with end-stage disease. ${ }^{11}$

Congestive cardiac failure appears to characterise the terminal stages of untreated Whipple's disease. In addition, it is estimated that the frequency of sudden death in Whipple's disease is around $20 \%{ }^{12}$ and such events are attributed to heart conduction defects and arrhythmias. Conduction tissue defects ${ }^{9}$ and various ECG changes including bundle branch block, ${ }^{13,14}$ complete heart block ${ }^{10}$ and non-specific ST/T wave changes ${ }^{4}$ have been documented.

Intrinsic heart muscle disease has never previously been described in Whipple's disease, although focal myocardial fibrosis has been observed in post mortem heart specimens. ${ }^{11}$ Moreover, heart failure as a presenting clinical feature, in the absence of significant valvular or pericardial disease, has not been previously documented. We have presented a unique case of Whipple's disease who developed florid cardiac failure in association with an atrial tachycardia and bundle branch block. Echocardiography showed four chamber dilatation and endomyocardial biopsy confirmed dilated heart muscle disease, with normal coronary arteries, never previously diagnosed in life in Whipple's disease.

This case acts as a reminder that Whipple's disease can present without gastrointestinal symptoms. The diagnosis should also be considered in the differential diagnosis of every case of dilated cardiomyopathy where there are associated features such as malabsorption, polyarthralgia, pigmentation, or neuropsychiatric disturbance.

We thank Dr S Singh for performing the endomyocardial biopsy and Dr J Gearty for assistance in interpretation of the small bowel histopathology.

3 Upton AC, Histochemical investigation of mesenchymal lesions in Whipple's disease. Am $\mathcal{F}$ Clin Pathol 1952; 22 755-64.

4 Pelech T, Fric P, Huslarova A, Jirasek A. Intestinal lymphocytic myocarditis in Whipple's disease. Lancet 1991; 337: 553-4. 
5 Trier JS, Phelps JC, Eidelman S, Rubin CE. Whipple's disease. Gastroenterology 1965; 48: 384-407.

6 Krauntz RF. Whipple's disease with cardiac and renal abnormalities. Arch Intern Med 1969; 123: 701-6.

7 Lie JT, Davis JS. Pancarditis in Whipple's disease: electron7 Lie JT, Davis JS. Pancarditis in Whipple's disease: electronAm f Clin Pathol 1976; 66: 22-30.

8 Enzinger FM, Helwig EB. Whipple's disease. A review of the literature and report of fifteen patients. Virchows Arch (A) 1963; 336: 238-69.

9 James TN, Haubrich WS. Bacterial arteritis in Whipple's disease. Circulation 1975; 52: 722-31.

10 Southern JF, Moscicki RA, Magro C, Dickersin GR, Fallon
JT, Bloch KJ. Lyphoedema, lymphocytic myocarditis, and sarcoidlike granulomatosis: manifestations of Whipple's disease $7 A M A$ 1989; 261: 1467-70.

11 McAllister HA, Fenoglio JJ. Cardiac involvement in Whipple's disease. Circulation 1975; 52: 152-6.

12 Rose AG. Mital stenosis in Whipple's disease. Thorax 1978; 33: $500-3$.

13 Fleming JL, Wiesner RH, Shorter RG. Whipple's disease: clinical, biochemical, and histopathological features and assessment of treatment in 29 patients. Mayo Clin Proc 1988; 63: 539-51.

14 Sossai P, DeBoni M, Cielo R. The heart and Whipple's disease. Int f Cardiol 1989; 23: 275-6.

\section{Central pontine myelinolysis following orthotopic liver transplant: association with cyclosporine toxicity}

Mustafa H Kabeer, Ronald S Filo, Martin L Milgrom, Mark D Pescovitz, Stephen B Leapman, Lawrence Lumeng, Rahul M Jindal

\begin{abstract}
Summary
Central pontine myelinolysis can occur after orthotopic liver transplantation leading to high mortality and serious morbidity. In our case, central pontine myelinolysis was associated with wide fluctuations in cyclosporine levels during an episode of hypocholesterolaemia, which may have precipitated central pontine myelinolysis.
\end{abstract}

Keywords: central pontine myelinolysis, liver transplantation, cyclosporine

\section{Introduction}

Central pontine myelinolysis is a distinctive lesion seen after a variety of conditions (box 1). ${ }^{1-3}$ With the widespread application of orthotopic liver transplantation, neurological complications such as central pontine myelinolysis has again come into focus. ${ }^{4}$

Since the introduction of cyclosporine and the new immunosuppressant FK506, neurological toxicity has been increasingly recognised as a complication of both therapies (box 2). Also reported are instances of akinetic mutism, severe dyskinesia and pseudo-bulbar

\begin{tabular}{|l|}
\hline Central pontine myelinolysis: \\
causes \\
\hline - rapid correction of sodium and osmolality in \\
patients who have underlying liver disease \\
- malnutrition \\
- alcoholism \\
- pneumonia \\
- lymphoma malignancies \\
- severe burns \\
\hline
\end{tabular}

palsies. ${ }^{7}$ It has been shown that cyclosporine neurotoxicity is specifically aggravated by low magnesium and low cholesterol levels. ${ }^{8}$

We report a case of central pontine myelinolysis after orthotopic liver transplantation in which high cyclosporine levels may have led to seizure activity, and central routine myelinolysis subsequently developed due to re-initiation of cyclosporine during an episode of hypocholesterolaemia.

\section{Case report}

A 50-year-old white man with end-stage liver disease secondary to cryptogenic cirrhosis was transplanted uneventfully with an ABOcompatible liver. During surgery there was no evidence of haemodynamic disturbances such as excessive blood loss or re-perfusion syndrome. Neurologic examination before transplantation was normal. His immunosuppressive protocol consisted of Imuran (iv $2 \mathrm{mg} / \mathrm{kg}$ ), methylprednisolone (iv $1 \mathrm{mg} / \mathrm{kg}$ ) and iv cyclosporine ( $2 \mathrm{mg} / \mathrm{kg}$ by continuous infusion) and switched to po medication when tolerated. He was extubated on the second day and was awake and neurologically stable, as were his other systems.

\begin{tabular}{|l|}
\hline Neurological complications of \\
cyclosporine toxicity \\
\hline - headache \\
- tremors \\
- convulsions \\
- coma \\
- quadria \\
- cerebral blindness \\
\hline
\end{tabular}

Box 2 